\title{
Physical Quantities of Reissner-Nordström Spacetime with Arbitrary Function and Regularized Procedure
}

\author{
Gamal G. L. Nashed ${ }^{1,2}$ \\ ${ }^{1}$ Center for Theoretical Physics, British University in Egypt, Sherouk City 11837, P.O. Box 43, Cairo, Egypt \\ ${ }^{2}$ Mathematics Department, Faculty of Science, Ain Shams University, Cairo 11566, Egypt \\ Correspondence should be addressed to Gamal G. L. Nashed; nashed@bue.edu.eg
}

Received 9 October 2012; Revised 26 April 2013; Accepted 2 May 2013

Academic Editor: Chao-Qiang Geng

Copyright (c) 2013 Gamal G. L. Nashed. This is an open access article distributed under the Creative Commons Attribution License, which permits unrestricted use, distribution, and reproduction in any medium, provided the original work is properly cited.

\begin{abstract}
We use the covariant teleparallel approach to compute the total energy of a spherically symmetric frame with an arbitrary function, that is, $\mathfrak{I}(r)$. We show how the total energy is always effected by the inertia. When use is made of the pure gauge connection, teleparallel gravity always yields the physically relevant result. We also calculate the total conserved charge and show how inertia spoils the physics in the time coordinate direction. Therefore, a regularized expression is employed to get a plausible value of energy. Finally, we use the Euclidean continuation method, in the context of TEGR, to calculate the energy, Hawking temperature, entropy, and first law of thermodynamics.
\end{abstract}

\section{Introduction}

Teleparallel theories are interesting for several reasons: first of all, general relativity (GR) can be considered as a particular theory of teleparallelism, and thus it could be viewed from a different viewpoint that can lead to the same results [1]. Second, one can define an energy-momentum tensor for the gravitational field that is a true tensor under all general coordinate transformations but not under local Lorentz transformation. This is the reason why teleparallelism was reconsidered by Møller when he was studying the problem of defining an energy-momentum tensor for the gravitational field $[2,3]$, but he did not succeed in defining a true tensor that is invariant under general coordinate transformations. The idea was taken over by Pellegrini and Plebański that constructed the general Lagrangian for these theories [4]. The third reason why these theories are interesting is that they can be seen as gauge theories of the translation group (not the full Poincaré group), and, thus, they give an alternative interpretation of GR [5-16].

Geometrically, teleparallel models are described by the Weitzenböck spacetime that is characterized by vanishing curvature and nonzero torsion. The tetrad (or a frame) is the basic field variable which can be treated as a gauge potential corresponding to the group of local translations.
Then, torsion is interpreted as the corresponding gauge field strength. As a result, a gravitational teleparallel Lagrangian is straightforwardly constructed from the quadratic torsion invariants.

Mathematically, there are infinitely many tetrads since a reference frame of an observer can obviously be constructed in an infinitely many ways. In particular, from a given tetrad field $h_{i}{ }^{\mu}$, we can obtain a continuous family of tetrads by performing the local Lorentz transformation $h_{i}^{\prime \alpha}=\Lambda^{\alpha}{ }_{\beta} h_{i}{ }^{\beta}$, where the elements of the Lorentz matrix $\Lambda^{\alpha}{ }_{\beta}(x)$ are arbitrary functions of the spacetime coordinates [17].

An important difference between Einstein theory and teleparallel theories is that it is possible to distinguish gravitational field from inertia [18]. With the purpose of getting a deeper insight into the covariant teleparallel formalism, Lucas et al. [17] reanalyze the computation of the total energy of two models. Obukhov et al. [19] computed the energy and momentum transported by exact plane gravitationalwave solutions of Einstein equations using the teleparallel equivalent formulation of Einstein's theory.

The existence of symmetries in a physical system means that there exist one or more transformations that leave the physical system invariant. Since physical systems can be fully described by their Lagrangians/Hamiltonians, it is natural to expect the symmetry transformations to be canonical 
ones. The number of symmetry transformations that can be generated from the system depends on how many conserved quantities of the associated system are considered. It has been known that conserved quantities of a physical system are linked to the generator of the associated symmetry transformations [20-22].

A number of methods for calculating energy and conserved charges that provide reliable result, are used in the present time. Using the Lagrange-Noether approach, one can derive the conserved currents that arise from the invariance of the classical action under transformations of fields. However, in Riemannian geometry, one cannot find symmetries that can be used to generate the conserved energy-momentum currents. Only one can speak about the energy of asymptotically flat spacetime. Earlier analysis of this problem can be found in details in $[23,24]$. In the framework of the TEGR, it is possible to make definite statements about the energy and momentum of the gravitational field. This fact constitutes the major motivation for considering this theory. In the $3+1$ formulation of the TEGR [25], and by imposing Schwinger's time gauge condition [26] for the tetrad field by fixing $h_{(a)}{ }^{0}=0$ which implies $h_{a}^{(0)}=$ 0 , it is found that the Hamiltonian and vector constraints contain each one a divergence in the form of scalar and vector densities, respectively, that can be identified with the energy and momentum densities of the gravitational field [27, 28]. Gibbons et al. [29] compute the energy of KerrAdS black holes first indirectly through integrating the first law and then using the conformal definition of Ashtekar et al. $[30,31]$, and they indicate that both of them agree. In addition to the Regge-Teitelboim method [32], some other various definitions are the following: the approach of Abbott and Deser [33-35], the spinor definition [36, 37] based on the electric Weyl tensor, covariant phase space methods [3841], cohomological techniques [42, 43], the KBL approach [44-46], Noether methods [47-52], and the "counter term subtraction method" $[53,54]$ (and more references on this in [40], for example) improved surface integrals [55] and regularization of the Euclidean action $[56,57]$.

Recently, the definition of conserved currents and charges for models with "quasi-invariant" Lagrangians is applied for Kerr-NUT spacetimes [58]. Within the framework of MAG, a stationary axially symmetric exact solution of the vacuum field equations is obtained for a specific gravitational Lagrangian ([59] and the references therein). In the teleparallel gravity, Nester et al. [60] have considered the quasilocal center of mass (COM). They have used the covariant Hamiltonian formalism, in which quasilocal quantities are given by the Hamiltonian boundary term, along with the covariant asymptotic Hamiltonian boundary expressions. Consideration of the COM not only gives the most restrictive asymptotic conditions on the variables but also gives strong constraints on the acceptable expressions [61]. A relation between spinor Lagrangian and teleparallel theory is established [61]. In metric-affine generalization of teleparallelism, it has been shown that there is an inconsistency in the coupling of spinors. Many discussions have been given for this in [62]. It's the aim of the present study to calculate energy, conserved charges, and thermodynamical quantities of a spherically symmetric frame with an arbitrary function. This calculation is done using two different procedures. In the first one, only Riemannian connection is considered, $\widetilde{\Gamma}_{\alpha}^{\beta}$, while in the second procedure, Weitzenböck connection $\Gamma_{\alpha}{ }^{\beta}$ is taken into account besides the Riemannian connection.

In Section 2, we use the language of exterior forms to give an outline of the teleparallel approach. In Section 3, we calculate the total energy of the spherically symmetric model and show that it is affected by inertia. Therefore, we use the covariant formalism and show that the Weitzenböck connection acts as a regularizing tool and always provides the physically meaningful result. In Section 4, we calculate the total conserved charge and show how inertia affects the final result. In Section 5, we use the regularized expression of conserved charge to recalculate the total charge, and plausible result is obtained. In Section 6, we calculate the energy using the Euclidean continuation method and also calculate Hawking temperature, entropy, and first law of thermodynamics. The final section is devoted for the main results and discussion.

Notation. The Latin indices $i, j, \ldots$ for local holonomic spacetime coordinates and the Greek indices $\alpha, \beta, \ldots$ label (co)frame components are used. Particular frame components are denoted by hats, $\widehat{0}, \widehat{1}$, and so forth. As usual, the exterior product is denoted by $\wedge$, while the interior product of a vector $\xi$ and a $p$-form $\Psi$ is denoted by $\xi\rfloor \Psi$. The vector basis dual to the frame 1-forms $\vartheta^{\alpha}$ is denoted by $e_{\alpha}$, and they satisfy $\left.e_{\alpha}\right\rfloor \vartheta^{\beta}=\delta_{\alpha}{ }^{\beta}$. Using local coordinates $x^{i}$, we have $\vartheta^{\alpha}=h_{i}^{\alpha} d x^{i}$ and $e_{\alpha}=h_{\alpha}^{i} \partial_{i}$, where $h_{i}^{\alpha}$ and $h_{\alpha}^{i}$ are the covariant and contravariant components of the tetrad field. We define the volume 4-form by $\eta=\vartheta^{\widehat{0}} \wedge \vartheta^{\widehat{1}} \wedge \vartheta^{\hat{2}} \wedge \vartheta^{\widehat{3}}$. Furthermore, with the help of the interior product, we define

$$
\left.\eta_{\alpha}=e_{\alpha}\right\rfloor \eta=\frac{1}{3 !} \epsilon_{\alpha \beta \gamma \delta} \vartheta^{\beta} \wedge \vartheta^{\gamma} \wedge \vartheta^{\delta}
$$

where $\epsilon_{\alpha \beta \gamma \delta}$ is completely antisymmetric with $\epsilon_{0123}=1$.

Consider

$$
\begin{gathered}
\left.\eta_{\alpha \beta}=e_{\beta}\right\rfloor \eta_{\alpha}=\frac{1}{2 !} \epsilon_{\alpha \beta \gamma \delta} \vartheta^{\gamma} \wedge \vartheta^{\delta}, \\
\left.\eta_{\alpha \beta \gamma}=e_{\gamma}\right\rfloor \eta_{\alpha \beta}=\frac{1}{1 !} \epsilon_{\alpha \beta \gamma \delta} \vartheta^{\delta},
\end{gathered}
$$

which are bases for 3-, 2-, and 1-forms, respectively. Finally,

$$
\left.\left.\left.\left.\left.\eta_{\alpha \beta \mu \nu}=e_{\nu}\right\rfloor \eta_{\alpha \beta \mu}=e_{\nu}\right\rfloor e_{\mu}\right\rfloor e_{\beta}\right\rfloor e_{\alpha}\right\rfloor \eta=\epsilon_{\alpha \beta \gamma \delta}
$$

is the Levi-Civita tensor density. The $\eta$-forms satisfy the useful identities:

$$
\begin{gathered}
\vartheta^{\beta} \wedge \eta_{\alpha}=\delta_{\alpha}^{\beta} \eta, \quad \vartheta^{\beta} \wedge \eta_{\mu \nu}=\delta_{\nu}^{\beta} \eta_{\mu}-\delta_{\mu}^{\beta} \eta_{\nu}, \\
\vartheta^{\beta} \wedge \eta_{\alpha \mu \nu}=\delta_{\alpha}^{\beta} \eta_{\mu \nu}+\delta_{\mu}^{\beta} \eta_{\nu \alpha}+\delta_{\nu}^{\beta} \eta_{\alpha \mu}, \\
\vartheta^{\beta} \wedge \eta_{\alpha \gamma \mu \nu}=\delta_{\nu}^{\beta} \eta_{\alpha \gamma \mu}-\delta_{\mu}^{\beta} \eta_{\alpha \gamma \nu}+\delta_{\gamma}^{\beta} \eta_{\alpha \mu \nu}-\delta_{\alpha}^{\beta} \eta_{\gamma \mu \nu} .
\end{gathered}
$$

The line element $d s^{2}=g_{\alpha \beta} \vartheta^{\alpha} \otimes \vartheta^{\beta}$ is defined by the spacetime metric $g_{\alpha \beta}$. 


\section{Brief Review of Teleparallel Gravity and Conserved Currents}

Teleparallel geometry can be viewed as a gauge theory of translation [63-68]. The coframe $\vartheta^{\alpha}$ plays the role of the gauge translational potential of the gravitational field. GR can be reformulated as the teleparallel theory. Geometrically, teleparallel gravity can be considered as a special case of the metric-affine gravity in which $\vartheta^{\alpha}$ and the local Lorentz connection are subject to the distant parallelism constraint $R_{\alpha}{ }^{\beta}=0$ [69-79]. In this geometry, the torsion 2-form

$$
\begin{aligned}
T^{\alpha} & =D \vartheta^{\alpha}=d \vartheta^{\alpha}+\Gamma_{\beta}{ }^{\alpha} \wedge \vartheta^{\beta}=\frac{1}{2} T_{\mu \nu}{ }^{\alpha} \vartheta^{\mu} \wedge \vartheta^{\nu} \\
& =\frac{1}{2} T_{i j}{ }^{\alpha} d x^{i} \wedge d x^{j}
\end{aligned}
$$

arises as the gravitational gauge field strength, $\Gamma_{\alpha}{ }^{\beta}$ being the Weitzenböck 1-form connection, $d$ is the exterior derivative, and $D$ is the exterior covariant derivative. The torsion $T^{\alpha}$ can be decomposed into three irreducible pieces [17]: the tensor part, the trace, and the axial trace given, respectively, by

$$
\begin{gathered}
{ }^{(1)} T^{\alpha}=T^{\alpha}-^{(2)} T^{\alpha}-^{(3)} T^{\alpha}, \quad \text { with } \\
\left.{ }^{(2)} T^{\alpha}=\frac{1}{3} \vartheta^{\alpha} \wedge T, \quad \text { where } T=\left(e_{\beta}\right\rfloor T^{\beta}\right), \\
\left.e_{\alpha}\right\rfloor T=T_{\mu \alpha}{ }^{\mu} \text {, polar trace of torsion } \\
\left.{ }^{(3)} T^{\alpha}=\frac{1}{3} e^{\alpha}\right\rfloor P, \quad \text { with } P=\left(\vartheta^{\beta} \wedge T_{\beta}\right), \\
\left.e_{\alpha}\right\rfloor P=T^{\mu \nu \lambda} \eta_{\mu \nu \lambda \alpha}, \text { axial trace of torsion. }
\end{gathered}
$$

The Lagrangian of the teleparallel equivalent of general relativity (TEGR) has the form (The effect of adding the non-Riemannian parity odd pseudoscalar curvature to the Hilbert-Einstein-Cartan scalar curvature was studied by many authors (cf. [80-84] and the references therein.))

$$
V=-\frac{1}{2 \kappa} T^{\alpha} \wedge^{*}\left({ }^{(1)} T_{\alpha}-2^{(2)} T_{\alpha}-\frac{1}{2}{ }^{(3)} T_{\alpha}\right) .
$$

$\kappa=8 \pi G / c^{3}, G$ is the Newtonian constant, $c$ is the speed of light, and $*$ denotes the Hodge duality in the metric $g_{\alpha \beta}$ which is assumed to be flat Minkowski metric $g_{\alpha \beta}=o_{\alpha \beta}=$ $\operatorname{diag}(+1,-1,-1,-1)$ that is used to raise and lower local frame (Greek) indices.

The variation of the total action with respect to the coframe which gives the field equations in the form

$$
D \widetilde{H}_{\alpha}-E_{\alpha}=\Sigma_{\alpha}, \quad \text { where } \Sigma_{\alpha}=\frac{\delta L_{\text {matter }}}{\delta \vartheta^{\alpha}}
$$

is the symmetric energy-momentum tensor 3-form of matter which is considered as the source. In accordance with the general Lagrange-Noether scheme $[55,69]$, one derives from (7) the translational momentum 2-form and the canonical energy-momentum 3-form (In the absence of Weitzenböck connection, the contortion $K_{\beta}^{\alpha}$ will be identical with Riemannian connection.)

$$
\begin{aligned}
\widetilde{H}_{\alpha} & =-\frac{\partial V}{\partial T^{\alpha}}=\frac{1}{\kappa} *\left({ }^{(1)} T_{\alpha}-2^{(2)} T_{\alpha}-\frac{1}{2}{ }^{(3)} T_{\alpha}\right) \\
& =\frac{1}{2 \kappa} \widetilde{\Gamma}^{\beta \gamma} \wedge \eta_{\alpha \beta \gamma}, \\
E_{\alpha} & \left.\left.=\frac{\partial V}{\partial \vartheta^{\alpha}}=e_{\alpha}\right\rfloor V+\left(e_{\alpha}\right\rfloor T^{\beta}\right) \wedge \widetilde{H}_{\beta} \\
& \left.\left.=\frac{1}{2}\left[\left(e_{\alpha}\right\rfloor d \vartheta^{\beta}\right) \wedge \widetilde{H}_{\beta}-d \vartheta^{\beta} \wedge\left(e_{\alpha}\right\rfloor \widetilde{H}_{\beta}\right)\right],
\end{aligned}
$$

where $K^{\beta \gamma}$ is the contortion 1-form which is related to the torsion through the relation

$$
T^{\alpha}=K_{\beta}^{\alpha} \wedge \vartheta^{\beta} .
$$

Due to geometric identities [17], the Lagrangian (7) can be recast as

$$
V=-\frac{1}{2} T^{\alpha} \wedge \widetilde{H}_{\alpha}
$$

The presence of the connection field $\Gamma_{\alpha}{ }^{\beta}$ plays an important regularizing role due to the following.

First. The theory becomes explicitly covariant under the local Lorentz transformations of the coframe; that is, the Lagrangian (7) is invariant under the change of variables

$$
\vartheta^{\prime \alpha}=\Lambda_{\beta}^{\alpha} \vartheta^{\beta}, \quad \Gamma_{\alpha}^{\prime \beta}=\left(\Lambda^{-1}\right)^{\mu}{ }_{\alpha} \Gamma_{\mu}{ }^{\gamma} \Lambda^{\beta}{ }_{\nu}+\Lambda_{\gamma}^{\beta} d\left(\Lambda^{-1}\right)_{\alpha}^{\gamma} .
$$

Due to the noncovariant transformation law of $\Gamma_{\alpha}{ }^{\beta}$, see (13), if a connection vanishes in a given frame, it will not vanish in any other frame related to the first by a local Lorentz transformation. When $\Gamma_{\alpha}{ }^{\beta}=0$ for all frames, which is the tetrad gravity, the Lagrangian (7) is only quasi-invariant; that is, it changes by a total divergence.

Second. $\Gamma_{\alpha}{ }^{\beta}$ plays an important role in the teleparallel framework. This role represents the inertial effects which arise from the choice of the reference system [17]. This means that when one changes the reference system, that is, uses different tetrad which reproduced the same metric, the effect of the inertia will change. The contributions of this inertial in many cases lead to unphysical results for the total energy of the system. Therefore, the role of the teleparallel connection is to separate the inertial contribution from the truly gravitational one. Since the teleparallel curvature is zero, the connection is a "pure gauge," that is,

$$
\Gamma_{\alpha}^{\beta} \stackrel{\text { def. }}{=}\left(\Lambda_{\gamma}^{\beta}\right)^{-1} d\left(\Lambda_{\alpha}^{\gamma}\right)
$$

The Weitzenböck connection always has the form (14). The relation between Weitzenböck and Riemannian connections has the form $[17,19]$

$$
\Gamma_{\alpha}^{\beta}=\widetilde{\Gamma}_{\alpha}^{\beta}-K_{\alpha}^{\beta}
$$


The teleparallel model (7) belongs to the class of quasiinvariant theories. In fact, one can verify that under a change of the coframe $\vartheta^{\prime \alpha}=\left(\Lambda(x)^{\alpha}{ }_{\beta}\right) \vartheta^{\beta}$, the Lagrangian changes by a total derivative:

$$
\widetilde{V}\left(\vartheta^{\prime}\right)=\widetilde{V}(\vartheta)-\frac{1}{2 \kappa} d\left[\left(\Lambda^{-1}\right)_{\beta}^{\alpha} d \Lambda_{\gamma}^{\beta} \wedge \eta_{\alpha}^{\gamma}\right]
$$

In addition to (16), it is easy to verify that (9) and (10) change like

$$
\begin{aligned}
\widetilde{H}_{\alpha}^{\prime}\left(\vartheta^{\prime}\right)= & \left(\Lambda^{-1}\right)_{\alpha}^{\beta} \widetilde{H}_{\beta}(\vartheta) \\
& -\frac{1}{2 \kappa}\left[\left(\Lambda^{-1}\right)_{\alpha}^{\beta}\left(\Lambda^{-1}\right)_{\gamma}^{\nu} d \Lambda^{\gamma}{ }_{\mu} \wedge \eta_{\beta}{ }^{\mu}{ }_{\nu}\right], \\
\widetilde{E}_{\alpha}^{\prime}\left(\vartheta^{\prime}\right)= & \left(\Lambda^{-1}\right)_{\alpha}^{\beta} \widetilde{E}_{\beta}(\vartheta)+d\left(\Lambda^{-1}\right)_{\alpha}^{\beta} \wedge \widetilde{H}_{\beta}(\vartheta) \\
& -\frac{1}{2 \kappa} d\left[\left(\Lambda^{-1}\right)_{\alpha}^{\beta}\left(\Lambda^{-1}\right)_{\gamma}^{\nu} d \Lambda^{\gamma}{ }_{\mu} \wedge \eta_{\beta}{ }^{\mu}{ }_{\nu}\right] .
\end{aligned}
$$

The total conserved charge in the tetrad gravity theory is given by [61]

$$
Q(\xi, \vartheta)=\int_{\partial S} \xi^{\alpha} \widetilde{H}_{\alpha}=\frac{1}{2 \kappa} \int_{\partial S} \xi^{\alpha} \widetilde{\Gamma}^{\beta \gamma} \wedge \eta_{\alpha \beta \gamma}
$$

where $\left.\xi^{\alpha}=\xi\right\rfloor \vartheta^{\alpha}$. Equation (18) is obviously reference frame dependent like Freud's superpotential for the Einstein pseudotensor [85]. Under a local Lorentz transformation, (18) transforms as

$$
\begin{aligned}
\widetilde{Q}\left(\xi, \vartheta^{\prime}\right) & =\widetilde{Q}(\xi, \vartheta)-\frac{1}{2 \kappa} \int_{\partial S} \xi^{\alpha}\left(\Lambda^{-1}\right)_{\gamma}^{\nu} d \Lambda_{\mu}^{\gamma} \wedge \eta_{\alpha}{ }^{\mu}{ }_{\nu} \\
& =\frac{1}{2 \kappa} \int_{\partial S} \xi^{\alpha}\left(\widetilde{\Gamma}^{\beta \gamma}-\Gamma^{\beta \gamma}\right) \wedge \eta_{\alpha \beta \gamma},
\end{aligned}
$$

where $\Gamma_{\mu}{ }^{\nu}$ is given by (14). The vector $\xi=\zeta^{i} \partial_{i}$ does not depend on the choice of the frame, while its components, that is, $\xi^{\alpha}$, transform as a vector. Equation (19) which depends on a tensorial object (the difference between 2 connections) is more satisfactory. In this study, we call it regularization but in the quasilocal literature it is described as a choice of [86].

\section{Reissner-Nordström Spacetime with Arbitrary Function}

In the spherical local coordinates $(t, r, \theta, \phi)$, a spherically symmetric spacetime with arbitrary function is described by [87]

$$
h^{\alpha}{ }_{i}=\left(\begin{array}{cccc}
\frac{\beta}{\alpha_{1}} & \beta_{1} \alpha_{1} \sin \theta \cos \phi & \beta_{1} r \sin \theta \sin \phi & \beta_{1} r \alpha_{1} \sin \theta \cos \theta \\
\frac{-\beta_{1}}{\alpha_{1}} f(\theta, \phi) & -\alpha_{1} \sin \theta \cos \phi\left[1+\beta_{2}\right] & -r\left[\sin \theta \sin \phi \beta_{2}+\cos \theta \cos \phi\right] & -r \sin \theta\left[\cos \theta \beta_{2}+\sin \phi\right] \\
\frac{-\beta_{1}}{\alpha_{1}} f_{1}(\theta, \phi) & -\alpha_{1} \sin \theta\left[\sin \phi+\cos \phi \beta_{3}\right] & -r \sin \phi\left[\sin \theta \beta_{3}+\cos \theta\right] & -r \sin \theta\left[\cos \theta \beta_{3}+\cos \phi\right] \\
\frac{\beta_{1}}{\alpha_{1}} f_{2}(\theta, \phi) & -\alpha_{1} \cos \theta\left[1+\tan \theta \cos \phi \beta_{4}\right] & -r \sin \theta\left[\sin \phi \beta_{4}+1\right] & r \sin \theta \cos \theta \beta_{4}
\end{array}\right),
$$

where $\alpha_{1}, \beta, \beta_{1}, \beta_{2}, \beta_{3}, \beta_{4}, f(r, \theta), f_{1}(r, \theta)$ and $f_{2}(r, \theta)$ have the form

$$
\begin{gathered}
\alpha_{1}=\left(1-\frac{2 M}{r}+\frac{q^{2}}{r^{2}}\right)^{-(1 / 2)}, \\
\beta=\left\{\frac{\left(r \mathfrak{I}^{\prime}-1\right)^{2}}{1-\left(2 c_{1} / r\right)+\left(c_{2}^{2} / r^{2}\right)}\right\}^{1 / 2}, \\
\beta_{1}=\sqrt{\beta^{2}-1,} \quad \beta_{2}=[\beta-1] f(\theta, \phi), \\
\beta_{3}=[\beta-1] f_{1}(\theta, \phi), \quad \beta_{4}=[\beta-1] f_{2}(\theta, \phi), \\
f(\theta, \phi)=\left(\sin ^{2} \theta \cos ^{2} \phi+\cos \theta \sin \phi[\sin \theta \cos \phi-1]\right), \\
f_{1}(\theta, \phi)=(\cos \theta \cos \phi+\sin \theta \sin \phi[\sin \theta \cos \phi+\cos \theta \sin \phi]), \\
f_{2}(\theta, \phi)=\sin \theta(\sin \theta \sin \phi-\cos \phi \cos \theta),
\end{gathered}
$$

where $c_{1}$ and $c_{2}$ are two constants and $M$ and $q$ are the mass and the electric charge parameters, respectively. The metric associated with tetrad (20) has the Reissner Nordström spacetime $[88,89]$. When $c_{2}=0$ and $q=0$, one returns to the Schwarzschild spacetime [90]. Tetrad (20) has been studied extensively in $[88,89]$ when $q=c_{1}=c_{2}=0$, but with different notation. In those studies, it is shown that the value of energy did not coincide with the ADM value [90, 91]. Some explanation related to the asymptotic behavior of $\mathfrak{\Im}$ has been given to explain how to make energy coincides with ADM [92]. Also, in [88, 89], it is shown that the value of energy in general depends on $\mathfrak{\Im}$. In the present study, it is our goal to recalculate the value of energy related to tetrad (20) using the method developed in [17]. It is of interest to note that any physical tetrad must behave as

$$
h_{\alpha i}=O_{\alpha i}+e_{\alpha i}\left(\frac{1}{r}\right),
$$

with $O_{\alpha i}$ as Minkowski's metric tensor and $e_{\alpha i}(1 / r)$ as the first term in the asymptotic expansion of $g_{\mu \nu}$. Asymptotically flat 
spacetimes are defined by the above unnumerated equation together with $\partial_{\beta} h_{\alpha i}=O\left(1 / r^{2}\right)$.

3.1. Calculation of Energy. The necessary component of the superpotential 2-form needed to the energy calculation takes the form (We will not write the nonvanishing components of Riemannian connection and Weitzenböck connection due to their huge form.):

$$
\begin{aligned}
\widetilde{H}_{\widehat{0}}=\frac{r \sin \theta}{8 \pi}[ & \left(1-\sin ^{2} \theta \cos ^{2} \phi\right. \\
& -\cos \theta \sin \phi[1+\sin \theta \cos \phi])\{\beta-1\} \\
& \left.+2\left\{1-\frac{\beta}{\alpha_{1}}\right\}\right](d \theta \wedge d \phi) .
\end{aligned}
$$

Computing the total energy at a fixed time in the 3-space with a spatial boundary 2-dimensional surface $\partial S=\{r=R, \theta, \phi\}$, we obtain

$$
\widetilde{E}=\int_{\partial S} \widetilde{H}_{\widehat{0}}=\frac{R}{3}\left\{2+\beta\left(1-\frac{3}{\alpha_{1}}\right)\right\} .
$$

From (24), when $\beta=1$, we obtain the value of energy up to $\left(1 / R^{2}\right)$ in the form:

$$
\begin{aligned}
\widetilde{E} & =R\left\{1-\frac{1}{\sqrt{1-(2 M / r)+\left(q^{2} / r^{2}\right)}}\right\} \\
& \approx M-\frac{q^{2}}{2 R}+\frac{3 M^{2}}{2 R}-\frac{3 M q^{2}-5 M^{3}}{2 R^{2}}+O\left(\frac{1}{R^{3}}\right)+\cdots
\end{aligned}
$$

Generally, (24) shows that the standard energy value for Reissner Nordström spacetime depends on the arbitrary function $\mathfrak{\Im}(r)$ and on the two constants $c_{1}$ and $c_{2}$. By giving a specific value of $\mathfrak{\Im}(r)$, we get a different value of the energy.

$$
\left(\Lambda_{2}{ }^{\gamma} \delta\right)=\left(\begin{array}{cc}
\beta & \beta_{1} \sin \theta \cos \phi \\
-\beta_{1} \sin \theta \cos \phi & 1+(1-\beta) \sin ^{2} \theta \cos ^{2} \phi \\
-\beta_{1} \sin \theta \sin \phi & (1-\beta) \sin ^{2} \theta \sin \phi \cos \phi \\
-\beta_{1} \cos \theta & (1-\beta) \sin \theta \cos \theta \cos \phi
\end{array}\right.
$$

$\left.\begin{array}{cc}\beta_{1} \sin \theta \sin \phi & \beta_{1} \cos \theta \\ (1-\beta) \sin ^{2} \theta \sin \phi \cos \phi & (1-\beta) \sin \theta \cos \theta \cos \phi \\ 1+(1-\beta) \sin ^{2} \theta \sin ^{2} \phi & (1-\beta) \sin \theta \cos \theta \sin \phi \\ (1-\beta) \sin \theta \cos \theta \sin \phi & 1+(1-\beta) \cos ^{2} \theta\end{array}\right)$.

Equation (28) in general is a boost transformation which depends on $\beta$, and $\left(\Lambda_{1}{ }^{\alpha} \gamma\right)$ is a special case of Euler's angle, and finally $\left(h^{\gamma}\right)_{R}$ is the square root of the Reissner-Nordström metric. The trivial case to undo the effect of $\beta$ is to put it equal 1. The coframe associated with tetrad (20) has the form

$$
\vartheta^{\alpha}=h^{\alpha}{ }_{i} d x^{i}=\left(\Lambda_{1}{ }_{\gamma}^{\alpha}\right)\left(\Lambda_{2}{ }^{\gamma}{ }_{\delta}\right) \vartheta_{R}^{\delta},
$$

where $\vartheta_{R}^{\delta}=\left(h^{\delta}{ }_{i}\right) d x^{i}$ has the form
3.2. Regularization of Energy. To overcome the contribution of inertia to physical quantities that appeared in the preceding subsection, we are going to use the regularization framework that is based on the covariance property, that is, taking into account the Weitzenböck connection $\Gamma_{\alpha}{ }^{\beta}$ provided by (14). The local Lorentz transformation introduced in [17] depends that is, depends on the radial coordinate $r$; however, in this study, we will introduce another local Lorentz transformation because the form of energy is not in the known form. If $\beta=1$, that is, $\mathfrak{J}(r)=$ constant and $c_{1}=c_{2}=q=0$, then tetrad (20) will coincide with tetrad (4.10) studied in [17] which gives the correct value of energy as shown after (24) and also in [17]. This means that the main reason for the contribution of inertia comes from the arbitrary function $\mathfrak{\Im}(r)$ and also from the two constants $c_{1}$ and $c_{2}$. Therefore, if we succeed in rewriting tetrad (20) as a composition of diagonal tetrad, local Lorentz transformation, which depends on the spatial coordinate, and another local Lorentz transformation, which depends on the arbitrary function, then we can use the resulting local Lorentz transformations as a regularizing tool that can remove inertia appearing in the previous subsection.

Tetrad (20) can be put into the following form:

$$
h_{i}^{\alpha}=\left(\Lambda_{1}^{\alpha} \gamma\right)\left(\Lambda_{2}{ }^{\gamma} \delta\right) h_{i}^{\delta}{ }_{i}
$$

where $\left(\Lambda_{1}{ }^{\alpha}{ }_{\gamma}\right)$ is a local Lorentz transformation given by

$$
\left(\Lambda_{1}{ }^{\alpha} \gamma\right)=\left(\begin{array}{cccc}
1 & 0 & 0 & 0 \\
0 & \sin \theta \cos \phi & \cos \theta \cos \phi & -\sin \phi \\
0 & \sin \theta \sin \phi & \cos \theta \sin \phi & \cos \phi \\
0 & \cos \theta & -\sin \theta & 0
\end{array}\right)
$$

and $\left(\Lambda_{2}{ }^{\gamma}{ }_{\delta}\right)$ is another local Lorentz transformation which has the form

$$
\begin{aligned}
\vartheta_{R}^{\widehat{0}} & =\frac{1}{\alpha_{1}} c d t, \quad \vartheta_{R}^{\hat{1}}=\alpha_{1} d r, \\
\vartheta_{R}^{\hat{2}} & =r d \theta, \quad \vartheta_{R}^{\widehat{3}}=r \sin \theta d \phi .
\end{aligned}
$$

The most appropriate form of local Lorentz transformation which is used in the calculation of the Weitzenböck connection, given by (14), is given by

$$
(\Lambda)^{\alpha}{ }_{\beta}=\left(\Lambda_{1}\right)_{\gamma}^{\alpha}\left(\Lambda_{2}{ }^{-1}\right)^{\gamma}{ }_{\delta}\left(\Lambda_{1}{ }^{-1}\right)_{\beta}^{\delta}, \quad \text { where }\left(\Lambda_{1}\right)_{\gamma}^{\alpha},\left(\Lambda_{2}\right)_{\gamma}^{\alpha}
$$
on the spatial coordinate because the energy is divergent, 
are defined in (27) and (28). This transformation is the only suitable one that can serve to remove the inertia and leave only the physical quantities as will be shown below. It is interesting to note that the use of the above equation is to return to the fact that Lagrangian (7) is not invariant under local Lorentz transformation as shown by (16). Due to this noninvariance, there exists an extra term which appears as shown by (17). To remove such term, the most suitable connection which help for such purpose is constructed out from the above equation. Calculating the necessary components of the superpotential 2-form, we finally get

$$
\widetilde{H}_{\widehat{0}}=\frac{r \beta \sin \theta\left(1-\left(1 / \alpha_{1}\right)\right)}{4 \pi}(d \theta \wedge d \phi) .
$$

Using (32) to compute the total energy at a fixed time in the 3 -space, with a spatial boundary 2-dimensional surface $\partial S=$ $\{r=R, \theta, \phi\}$, we finally obtain the asymptotic value of the energy up to $\left(1 / R^{2}\right)$ in the form

$$
\begin{aligned}
\widetilde{E} & =R \beta\left\{1-\frac{1}{\sqrt{1-(2 M / R)+\left(q^{2} / R^{2}\right)}}\right\} \\
& \approx M-\frac{q^{2}}{2 R}+\frac{3 M^{2}}{2 R}+\frac{c_{1} M}{R}+O\left(\frac{1}{R^{2}}\right)+\cdots,
\end{aligned}
$$

which is the value of energy of Reissner Nordström spacetime up to order $O(1 / R)[88,89,93,94]$. Equation (33) shows in a clear way that whatever the value of $\beta$, there will be no contribution to $O\left(1 / R^{0}\right)$. The contribution begins from $O(1 / R)$ as is clear from (33) when $\mathfrak{I} \sim$ const. or $\mathfrak{I} \sim 1 / R$, and so forth.

\section{Total Conserved Charge of Reissner-Nordström Spacetime with Arbitrary Function}

We are going to calculate the necessary quantities needed to compute the conserved charge. The nonvanishing components of Riemannian connection of tetrad (20), up to $O\left(1 / r^{3}\right)$ when $\beta=1$, have the form

$$
\begin{aligned}
\widetilde{\Gamma}_{\widehat{0}}^{\hat{1}} \approx-(M \sin \theta \cos \phi(1 & +2 \sin \phi \cos \theta \\
& \left.\times[1-\sin \theta \cos \phi]+2 \cos ^{2} \phi \sin ^{2} \theta\right) \\
\left.\times\left(r^{2}\right)^{-1}\right) d t & +O\left(\frac{1}{r^{3}}\right), \\
\widetilde{\Gamma}_{\widehat{0}}^{\hat{2}} \approx\left(M \operatorname { s i n } \theta \left(2 \sin \theta \cos \theta \cos \phi \sin ^{2} \phi\right.\right. & -\sin \phi\left[1-2 \cos ^{2} \phi \sin ^{2} \theta\right] \\
& \left.\left.+2 \cos \theta \cos ^{2} \phi\right) \times\left(r^{2}\right)^{-1}\right) d t+O\left(\frac{1}{r^{3}}\right),
\end{aligned}
$$

$$
\begin{aligned}
& \widetilde{\Gamma}_{\widehat{0}}^{\widehat{3}} \approx-( M\left(\cos \theta\left[1-2 \cos ^{2} \phi \sin ^{2} \theta\right]\right. \\
&+\left.2 \sin \theta \sin \phi \cos \phi \sin ^{2} \theta\right) \\
&\left.\times\left(r^{2}\right)^{-1}\right) d t+O\left(\frac{1}{r^{3}}\right) .
\end{aligned}
$$

The components of the vector $\xi^{\alpha}$ have the form

$$
\begin{aligned}
& \xi^{0}=-\frac{\zeta_{0} \sqrt{r^{2}-2 M r+q^{2}}}{\sqrt{r^{2}-2 c_{1} r+c_{2}^{2}}}, \\
& \xi^{1}=-\left(\left(\zeta_{0}\left(\cos \theta \sin \phi[1-\sin \theta \cos \phi]-\cos ^{2} \phi \sin ^{2} \theta\right)\right.\right. \\
& \left.\times\left(r^{2}-2 M r+q^{2}\right) \sqrt{2 c_{1} r-c_{2}^{2}}\right) \\
& \left.\times\left(r \sqrt{r^{2}-2 M r+q^{2}} \sqrt{r^{2}-2 c_{1} r+c_{2}^{2}}\right)^{-1}\right), \\
& \xi^{2}=-\left(\left(\zeta _ { 0 } \left(\cos \theta\left[\cos \phi+\sin \theta \sin ^{2} \phi\right]\right.\right.\right. \\
& \left.+\sin \phi \cos \phi \sin ^{2} \theta\right) \\
& \left.\times\left(r^{2}-2 M r+q^{2}\right) \sqrt{2 c_{1} r-c_{2}^{2}}\right) \\
& \left.\times\left(r \sqrt{r^{2}-2 M r+q^{2}} \sqrt{r^{2}-2 c_{1} r+c_{2}^{2}}\right)^{-1}\right), \\
& \xi^{3}=\left(\zeta_{0}(\sin \theta[\cos \theta \cos \phi-\sin \theta \sin \phi])\right. \\
& \left.\times\left(r^{2}-2 M r+q^{2}\right) \sqrt{2 c_{1} r-c_{2}^{2}}\right) \\
& \times\left(r \sqrt{r^{2}-2 M r+q^{2}} \sqrt{r^{2}-2 c_{1} r+c_{2}^{2}}\right)^{-1} .
\end{aligned}
$$

Finally, the nonvanishing components of the asymptotic form of the superpotential 2-form $\widetilde{H}_{\widehat{\alpha}}$ up to $O(1 / r)$ have the form

$$
\begin{aligned}
\widetilde{H}_{\hat{0}}=\frac{\sin \theta}{r}( & {\left[2 r c_{1}+3 c_{1}^{2}-c_{2}^{2}\right] } \\
& \times\left\{\cos ^{2} \phi \sin ^{2} \theta+\sin \phi \cos \theta[1+\sin \theta \cos \phi]\right\} \\
& +2 q^{2}-4 M\left(c_{1}-r\right)-2 M^{2} \\
& \left.-c_{2}{ }^{2}+c_{1}\left(3 c_{1}+2 r\right)\right)(d \theta \wedge d \phi)+\cdots
\end{aligned}
$$




$$
\begin{aligned}
\widetilde{H}_{\widehat{1}}=\frac{\sin \theta}{\sqrt{c_{1} r}}( & {\left[12 c_{1}\left(c_{1}+r\right)-8 M c_{1}-3 c_{2}^{2}\right] } \\
& \times \cos \phi \sin \theta\{\cos \phi \sin \theta+\cos \theta \sin \phi\} \\
& +\sin \phi \cos \theta\left[8 M c_{1}+c_{2}{ }^{2}-4 c_{1}\left(c_{1}+r\right)\right] \\
& \left.-4 c_{1}\left(c_{1}+r\right)+c_{2}^{2}\right)(d \theta \wedge d \phi)+\cdots, \\
\widetilde{H}_{\widehat{2}}=\frac{\sin \theta}{\sqrt{c_{1} r}}( & {\left[8 M c_{1}-12 c_{1}\left(c_{1}+r\right)-3 c_{2}^{2}\right] \cos \phi \sin \phi \sin ^{2} \theta } \\
& +\sin \theta \cos \theta \sin ^{2} \phi\left[8 M c+3 c_{2}^{2}-12 c_{1}\left(c_{1}+r\right)\right] \\
& \left.\times \cos \phi \cos \theta\left[8 M c_{1}-4 c_{1}\left(c_{1}+r\right)+c_{2}^{2}\right]\right) \\
\times(d \theta \wedge & d \phi)+\cdots, \\
\widetilde{H}_{\widehat{3}}=\frac{\sin \theta}{\sqrt{c_{1} r}}[ & \sin \phi\left[8 M c_{1}-4 c_{1}\left(c_{1}+r\right)+c_{2}^{2}\right] \\
& -\left[8 M c_{1}-12 c_{1}\left(c_{1}+r\right)+3 c_{2}^{2}\right] \\
& \times \cos \theta(\cos \theta \sin \phi+\sin \theta \cos \phi)](d \theta \wedge d \phi)
\end{aligned}
$$

Using (35) as well as (36) in (18), we finally get the total conserved charges, up to $O(1 / r)$, in the following form:

$$
Q=\int_{\partial S} \xi^{\alpha} \widetilde{H}_{\alpha} \approx\left(M+\frac{2 c_{1}}{3}-\frac{3 q^{2}+2 c_{2}^{2}}{6 r}\right) \zeta^{0}+O\left(\frac{1}{r}\right) .
$$

For the tetrad determined by (20), the conserved charge corresponding to the diffeomorphism generated by the shift along the time coordinate has a nonusual value of the total energy of the configuration.

\section{Regularization of Conserved Charge}

The regularized form of the total conserved charge which can solve the above problem has the form [71]

$$
\widetilde{Q}(\xi, \vartheta)=\frac{1}{2 \kappa} \int_{\partial S} \xi^{\alpha}\left(\widetilde{\Gamma}^{\beta \gamma}-\Gamma^{\beta \gamma}\right) \wedge \eta_{\alpha \beta \gamma}
$$

where $\Gamma^{\beta \gamma}$ has the form (13).

Again the most appropriate local Lorentz transformation which is used in the calculation of Weitzenböck connection has the form

$$
(\Lambda)^{\alpha}{ }_{\beta}=\left(\Lambda_{1}\right)_{\gamma}^{\alpha}\left(\Lambda_{2}{ }^{-1}\right)_{\delta}^{\gamma}\left(\Lambda_{1}{ }^{-1}\right)_{\beta}^{\delta} .
$$

Using the above data, that is, Riemannian connection and Weitzenböck connection in (38), we get up to $O(1 / r)$

$$
\begin{aligned}
\widetilde{Q}(\xi, \vartheta) & =\int_{\partial S} \xi^{\alpha}\left(\widetilde{\Gamma}^{\beta \gamma}-\Gamma^{\beta \gamma}\right) \wedge \eta_{\alpha \beta \gamma} \\
& =\left(M-\frac{q^{2}}{2 r}\right) \zeta^{0}+O\left(\frac{1}{r^{2}}\right) .
\end{aligned}
$$

Therefore, the last term in (38) improves the unusual coefficients in (37). It is of interest to note that the calculation of regularized total conserved charges is more accurate than the regularization of energy; this is due to the fact that the latter remove completely the contribution of inertia.

\section{Thermal Properties of Reissner-Nordström Spacetime with Arbitrary Function}

In GR, thermodynamical quantities are calculated using the Euclidean continuation of metric [95]. However, in TEGR, these quantities are calculated through the divergence term, which appears in the Lagrangian [90]. This term has no effect on the field equation.

Gibbons and Hawking [95] discussed the thermal properties of the Schwarzschild solution, for which the line element takes the positive-definite standard form

$$
d s^{2}=+\left(1-\frac{2 M}{r}\right) d \tau^{2}+\left(1-\frac{2 M}{r}\right)^{-1} d r^{2}+r^{2} d \Omega^{2}
$$

after Euclidean continuation of the time variable, $t=-i \tau$. By using the transformation $x=4 M(1-2 M / r)^{1 / 2}$, the line element squared becomes

$$
d s^{2}=+\left(\frac{x}{4 M}\right)^{2} d \tau^{2}+\left(\frac{r^{2}}{4 M^{2}}\right)^{2} d x^{2}+r^{2} d \Omega^{2}
$$

which shows that $\tau$ can be regarded as an angular variable with period $8 \pi M$. Now, the Euclidean section of the Schwarzschild solution is the region defined by $8 \pi M \geq$ $\tau \geq 0$ and $x>0$, where the metric is positive definite, asymptotically flat, and nonsingular. They calculated the Euclidean action, $\widehat{I}$, of GR from the surface term as

$$
\widehat{I}=4 \pi M^{2}=\frac{\chi^{2}}{16 \pi}
$$

where $\chi=8 \pi M=T^{-1}$ with $T$ being interpreted as the absolute temperature of the Schwarzschild black hole.

For a canonical ensemble, the energy is given by [95-97]

$$
E=\frac{\sum_{n} E_{n} e^{-\chi E_{n}}}{\sum_{n} e^{-\chi E_{n}}}=-\frac{\partial}{\partial \chi} \log Z,
$$


where $E_{n}$ is the energy in the $n$th state and $Z$ is the partition function, which is in the tree approximation related to the Euclidean action of the classical solution by

$$
\widehat{I}=-\log Z .
$$

Use of (43) and (45) in (44) gives

$$
E=\frac{\chi}{8 \pi}=M
$$

They also calculated the entropy of the Schwarzschild black hole to obtain

$$
S=-\sum_{n} P_{n} \log P_{n}=\chi E+\log Z=4 \pi M^{2}=\frac{1}{4} A,
$$

$$
\begin{gathered}
\left(h^{\alpha}\right)_{E}=\left\{\begin{array}{cc}
-\frac{\beta_{5}}{\alpha_{1}} & -\frac{\beta_{1}}{\alpha_{1}} \sin \theta \cos \phi \\
\frac{\beta_{1} f(\theta, \phi)}{\alpha_{1}} & \alpha_{1} \sin \theta \cos \phi\left[1+\beta_{6}\right] \\
\frac{\beta_{1} f_{1}(\theta, \phi)}{\alpha_{1}} & \alpha_{1} \sin \theta\left[\sin \phi+\cos \phi \beta_{7}\right] \\
-\frac{\beta_{1}}{\alpha_{1}} f_{2}(\theta, \phi) & -\alpha_{1} \cos \theta\left[1+\tan \theta \cos \phi \beta_{8}\right]
\end{array}\right. \\
\beta_{5}=\left\{2-\beta^{2}\right\}^{1 / 2}, \quad \beta_{6}=\left[1-\beta_{5}\right] f(\theta, \phi), \\
\beta_{7}=\left[1-\beta_{5}\right] f_{1}(\theta, \phi), \quad \beta_{8}=\left[1-\beta_{5}\right] f_{2}(\theta, \phi) .
\end{gathered}
$$

The Euclidean line element squared of tetrad (49) takes the form

$$
d s^{2}=\frac{1}{\alpha_{1}} d \tau^{2}+\alpha_{1} d r^{2}+r^{2}\left(d \theta^{2}+\sin ^{2} \theta d \phi^{2}\right),
$$

which is the Euclidian Reissner Nordström spacetime [88, 89]. Using (6), we get the following:

$$
h=r^{2} \sin \theta,
$$

$$
\begin{aligned}
& { }^{(2)} T^{1} \\
& \quad=((\beta+1)(1+\sin \theta \sin \phi[\cos \theta \cos \phi+\sin \theta \sin \phi] \\
& \left.\quad+\cos \theta \sin \phi)+r \alpha_{2}-\frac{2}{\alpha_{1}}\right) \\
& \quad \times\left(r \alpha_{1}\right)^{-1}, \quad \alpha_{2}=\alpha_{1}^{\prime},
\end{aligned}
$$

where $h=\sqrt{g}$ is the determinant of tetrad (49). There are other nonvanishing components of ${ }^{(2)} T^{\mu}$, that is, ${ }^{(2)} T^{0},{ }^{(2)} T^{2}$, and ${ }^{(2)} T^{3}$, but these components are not necessary in our computations. The volume integral (48), where $P_{n}=Z^{-1} e^{-\chi E_{n}}$ and $A$ is the area of the event horizon of the Schwarzschild black hole.

Now, let us apply Hawking and Gibbons procedure to tetrad (20). The Euclidean action is given by [90]

$$
\begin{aligned}
\widehat{I} & =-\frac{1}{2 \kappa} \int \sqrt{g}\left(R-2^{(2)} T_{; \mu}^{\mu}\right) d^{4} x \\
& =\frac{1}{\kappa} \int \sqrt{g}^{(2)} T_{; \mu}^{\mu} d^{4} x,
\end{aligned}
$$

where $R$ is the Riemann-Christoffel scalar curvature, which is vanishing for the Reissner Nordström spacetime, and ${ }^{(2)} T^{\mu}$ is the basic vector field of the torsion given by (6).

Tetrad (20) after using Euclidean continuation, $t=$ $-\sqrt{-1} \tau$, takes the following form:

$$
\left.\begin{array}{cc}
-\frac{\beta_{1}}{\alpha_{1}} \sin \theta \sin \phi & -\frac{\beta_{1}}{\alpha_{1}} \sin \theta \cos \theta \\
r\left[\sin \theta \sin \phi \beta_{6}+\cos \theta \cos \phi\right] & r \sin \theta\left[\cos \theta \beta_{6}+\sin \phi\right] \\
r \sin \phi\left[\sin \theta \beta_{7} \cos \theta\right] & r \sin \theta\left[\cos \theta \beta_{7}+\cos \phi\right] \\
-r \sin \theta\left[\sin \phi \beta_{8}+1\right] & -r \sin \theta \cos \theta \beta_{8}
\end{array}\right),
$$

$(\theta=0 \cdots \pi, \phi=0 \cdots 2 \pi, \tau=0 \cdots \chi)$, for tetrad (49), is calculated to give

$$
\begin{aligned}
\widehat{I} & =\frac{1}{\kappa} \int \sqrt{g}\left({ }^{(2)} T_{; \mu}^{\mu}\right) d^{4} x \\
& \approx \frac{2 \pi r^{2}\left(1+2 \beta-\left(6 / \alpha_{1}\right)-3 r \alpha_{2}\right)}{3 \alpha_{1}\left(1+\left(q^{2} / r^{2}\right)\right)},
\end{aligned}
$$

where $\chi=4 \pi r /\left(1-\left(q^{2} / r^{2}\right)\right)$. The largest positive root of $g^{r r}=0$, that is, $\left(r^{2}-2 M r+q^{2}\right)=0 \Rightarrow r_{ \pm}=M \pm \sqrt{M^{2}-q^{2}}$. Now, one can write mass and charge in terms of horizons, that is, $M=\left(r_{+}+r_{-}\right) / 2$ and $q=\sqrt{r_{-} r_{+}}$. Equation (53) shows in a clear way that the Euclidean action depends on the arbitrary function $\mathfrak{J}$ which makes the energy also depends on this arbitrary function. Therefore, we need to regularize (48). This regularization lies in the redefinition of the nonsymmetric connection. This redefinition takes the form

$$
\begin{gathered}
\Gamma_{j k}^{i} \stackrel{\text { def. }}{=} h_{\alpha}{ }^{i} h_{j, k}^{\alpha}+h_{\alpha}{ }^{i} \Lambda_{2}{ }^{\beta \alpha} \Lambda_{2 \beta \gamma, k} h^{\gamma}, \\
T^{\mu}{ }_{\nu \lambda} \stackrel{\text { def. }}{=} \Gamma^{\mu}{ }_{\nu \lambda}-\Gamma_{\lambda \nu}^{\mu}, \quad{ }^{(2)} T_{\nu} \stackrel{\text { def. }}{=} T^{\mu}{ }_{\nu \mu} .
\end{gathered}
$$

Using (54) in (48), we get

$$
\widehat{I}=\frac{1}{\mathcal{\kappa}} \int \sqrt{g}\left({ }^{(2)} T_{; \mu}^{\mu}\right) d^{4} x \approx \pi r_{+}{ }^{2}\left(1-\frac{q^{2}}{r_{+}{ }^{2}}\right) .
$$


TABLE 1: Comparison between the results of the arbitrary function $\mathfrak{\Im}(r)$, other constants, and the associated energy.

\begin{tabular}{|c|c|c|c|c|c|}
\hline Spacetime & Arbitrary function $B(r)$ & $\begin{array}{c}\text { The constants } \\
c_{1} \text { and } c_{2}\end{array}$ & Energy & Total conserved charge & $\begin{array}{l}\text { Regularized conserved } \\
\text { charge }\end{array}$ \\
\hline \multirow{3}{*}{ Schwarzschild } & $\mathfrak{J}(r)=0$ or & $\begin{array}{c}c_{1}=0 \\
\Im(r)=\text { const. }\end{array}$ & $\widetilde{E}=M$ & $\mathscr{Q}(\xi, \vartheta)=M \zeta^{0}$ & $\widetilde{Q}(\xi, \vartheta)=M \zeta^{0}$ \\
\hline & $\begin{array}{l}\mathfrak{I}(r)=0 \text { or } \\
\mathfrak{J}(r)=\text { const. }\end{array}$ & $c_{1}=M$ & $\widetilde{E}=\frac{M}{3}$ & $Q(\xi, \vartheta)=\frac{5 M}{3} \zeta^{0}$ & $\widetilde{Q}(\xi, \vartheta)=M \zeta^{0}$ \\
\hline & $\mathfrak{J}(r)=\int \frac{1-\sqrt{1-\left(2 c_{1} / r\right)}}{r} d r$ & $c_{1} \neq 0$ & $\widetilde{E}=M$ & $Q(\xi, \vartheta)=M \zeta^{0}$ & $\widetilde{\widetilde{Q}}(\xi, \vartheta)=M \zeta^{0}$ \\
\hline \multirow{3}{*}{$\begin{array}{l}\text { Reissner- } \\
\text { Nordström }\end{array}$} & $\begin{array}{l}\mathfrak{J}(r)=0 \text { or } \\
\mathfrak{J}(r)=\text { const. }\end{array}$ & $\begin{array}{c}c_{1}=0 \text { and } \\
c_{2}=0\end{array}$ & $\widetilde{E}=M-\frac{q^{2}}{2 R}$ & $\begin{array}{l}\mathbb{Q}(\xi, 9) \\
\approx\left(M-\frac{q^{2}+M^{2}}{2 r}\right) \zeta^{0} \\
\quad+O\left(\frac{1}{r^{2}}\right)\end{array}$ & $\begin{array}{l}\widetilde{Q}(\xi, \vartheta) \\
\approx\left(M-\frac{q^{2}+M^{2}}{2 r}\right) \zeta^{0} \\
\quad+O\left(\frac{1}{r^{2}}\right)\end{array}$ \\
\hline & $\begin{array}{l}\mathfrak{J}(r)=0 \text { or } \\
\mathfrak{I}(r)=\text { const. }\end{array}$ & $\begin{array}{c}c_{1}=M \text { and } \\
c_{2}=q\end{array}$ & $\begin{array}{l}\widetilde{E}= \\
\frac{1}{3}\left(M-\frac{q^{2}}{2 R}\right)\end{array}$ & $\begin{array}{l}\mathbb{Q}(\xi, \vartheta) \\
\approx\left(\frac{5 M}{3}-\frac{5 q^{2}+M^{2}}{6 r}\right) \zeta^{0} \\
\quad+O\left(\frac{1}{r^{2}}\right)\end{array}$ & $\begin{array}{l}\widetilde{\widetilde{Q}}(\xi, \vartheta) \\
\approx\left(M-\frac{q^{2}+M^{2}}{2 r}\right) \zeta^{0} \\
\quad+O\left(\frac{1}{r^{2}}\right)\end{array}$ \\
\hline & $\begin{array}{l}\mathfrak{I}(r)= \\
\int \frac{1-\sqrt{1-\left(2 c_{1} / r\right)+\left(c_{2}^{2} / r^{2}\right)}}{r} d r\end{array}$ & $\begin{array}{c}c_{1} \neq 0 \text { and } \\
c_{2} \neq 0\end{array}$ & $\widetilde{E}=M-\frac{q^{2}}{2 R}$ & $\begin{array}{l}\mathbb{Q}(\xi, \vartheta) \\
\approx\left(M-\frac{q^{2}+M^{2}}{2 r}\right) \zeta^{0} \\
\quad+O\left(\frac{1}{r^{2}}\right)\end{array}$ & $\begin{array}{l}\widetilde{\widetilde{Q}}(\xi, \vartheta) \\
\approx\left(M-\frac{q^{2}+M^{2}}{2 r}\right) \zeta^{0} \\
\quad+O\left(\frac{1}{r^{2}}\right)\end{array}$ \\
\hline
\end{tabular}

TABLE 2: Comparison between the results of energy using different translational momentum.

\begin{tabular}{|c|c|c|}
\hline Translational momentum & $\begin{array}{l}\text { Energy of Schwarzschild and Reissner-Nordström } \\
\text { spacetimes }\end{array}$ & Equation \\
\hline $\begin{array}{l}\text { Møller within the framework of tetrad } \\
\text { spacetime }\end{array}$ & $\begin{array}{l}\text { Energy of Schwarzschild spacetime } \\
\qquad E=2 M-R^{2} \mathscr{B}^{\prime}(\mathscr{R})\end{array}$ & (46) in [94] \\
\hline $\begin{array}{l}\text { Equation (9) with trivial } \\
\text { Weitzenböck connection, that is, } \Gamma_{\alpha}{ }^{\beta}=0 \text {, } \\
q=c_{2}=0\end{array}$ & $\begin{array}{l}\text { Energy of Schwarzschild spacetime } \\
\qquad E=\frac{R}{3}\left\{2+\beta\left(1-\frac{3}{\alpha_{1}}\right)\right\}\end{array}$ & $(24)$ \\
\hline $\begin{array}{l}\text { Equation (9) with nontrivial Weitzenböck } \\
\text { connection, that is, } \Gamma_{\alpha}{ }^{\beta} \neq 0, q=c_{1}=0\end{array}$ & $\begin{array}{l}\text { Energy of Schwarzschild spacetime } \\
\qquad E \approx M+O\left(\frac{1}{R}\right)\end{array}$ & (33) \\
\hline $\begin{array}{l}\text { Møller within the framework of tetrad } \\
\text { spacetime }\end{array}$ & $\begin{array}{l}\text { Energy of Reissner-Nordström spacetime } \\
\qquad E=2 M-\frac{q^{2}}{R}-R^{2} \mathfrak{I}^{\prime}(\mathscr{R})\end{array}$ & (38) in [93] \\
\hline $\begin{array}{l}\text { Equation (9) with trivial } \\
\text { Weitzenböck connection, that is, } \Gamma_{\alpha}^{\beta}=0\end{array}$ & $\begin{array}{l}\text { Energy of Reissner-Nordström spacetime } \\
\qquad E=\frac{R}{3}\left\{2+\beta\left(1-\frac{3}{\alpha_{1}}\right)\right\}\end{array}$ & $(24)$ \\
\hline $\begin{array}{l}\text { Equation (9) with nontrivial Weitzenböck } \\
\text { connection, that is, } \Gamma_{\alpha}^{\beta} \neq 0\end{array}$ & $\begin{array}{l}\text { Energy of Reissner-Nordström spacetime } \\
\qquad E \approx M-\frac{q^{2}}{2 R}+O\left(\frac{1}{R^{2}}\right)\end{array}$ & (33) \\
\hline
\end{tabular}

From (55), (44), and (45), we get the energy up to $O\left(1 / r_{+}\right)$in the form

$$
E \approx M-\frac{q^{2}}{2 r_{+}}+O\left(\frac{1}{{r_{+}}^{2}}\right)
$$

which is consistent with (33) and (40).
Using (55) and (56) in (47), we get the entropy of tetrad (49) in the form

$$
S \approx \pi r_{+}^{2}
$$

The Hawking temperature of tetrad (49) has the form [98]

$$
T=\frac{\left(1-\left(q^{2} / r_{+}^{2}\right)\right)}{4 \pi r_{+}} .
$$


From (56), (57), and (58), we get

$$
d M=T d S .
$$

Equation (59) shows that the first law of thermodynamic is satisfied.

\section{Discussion and Conclusion}

Within the framework of MAG, the total Lagrangian is formed from a geometrical part plus a matter part; that is, $\mathscr{L}_{\text {total }}=V\left(g_{\alpha \beta}, \vartheta^{\alpha}, Q_{\alpha \beta}, T^{\alpha}, R_{\alpha}{ }^{\beta}\right)+L_{\text {matter }}\left(g_{\alpha \beta}, \vartheta^{\alpha}, \Psi, D \Psi\right)$ with $Q_{\alpha \beta}$ is the nonmetricity, and $\Psi$ is the matter fields. Baekler and Hehl [59] have obtained a stationary axially symmetric exact solution of the vacuum field equation for a specific gravitational Lagrangian. Their black hole solution embodies a Kerr-de Sitter metric and the post-Riemannian structures of torsion and nonmetricity and is characterized by mass, angular momentum, and shear charge which measure the violation of Lorentz invariance. When the cosmological constant vanishes, that is, $\lambda=0$, the solution given by (57) derived in [59] will be identical with the third coframe defined by $\vartheta^{R}{ }_{3}^{\alpha}=\left(\Lambda_{1}{ }^{\alpha}{ }_{\gamma}\right)\left(h^{\gamma}{ }_{i}\right)_{3} d x^{i}$.

To test the regularizing property of teleparallelism, we have used the superpotential 2-form to calculate energy of $a$ spherically symmetric tetrad with arbitrary function. We have done such calculations when the Weitzenböck connection is taken into account and also when it is not taken into account. It has been shown that the energy depends on the arbitrary function in the trivial case and when it takes specific values, which make the local Lorentz transformations identical with the Kronecker delta; the total energy becomes physically acceptable [91, 94]. As Weitzenböck connection is taken into account and recalculation of energy is carried out, we have shown that it acts as a regularizing tool which separates inertia from the physical quantities.

In the context of TEGR, the total conserved charge of the spherically symmetric tetrad with arbitrary function is calculated. It has been shown how inertia spoils the total conserved charge along the time coordinate. Therefore, regularized expression to recalculate the total conserved charge is used, and a plausible result is obtained for energy. Finally, the energy of the tetrad field with arbitrary function is calculated using the Euclidean continuation method in the context of TEGR. It has been shown that energy depends on the arbitrary function. Therefore, a redefinition of the Riemannian connection is provided to recalculate energy. A satisfactory result which is consistent with the previous two methods is achieved. The thermodynamical quantities associated with the tetrad with arbitrary function are calculated, and satisfactory results are obtained [99]. The main conclusion of the present work is that Weitzenböck connection which acts as a regularized term works correctly for the spherically symmetric case. At present, it is not clear if this conclusion is correct for axially symmetric spacetimes. This needs a further study.

Blagojević [100] has assumed that spacetime behaves asymptotically as Minkowski space M4 and has obtained the corresponding global Poincaré generators. The surface terms related to the improved form of these generators appeared to represent the relevant conserved charges-energy, momentum, and angular momentum. Mielke and Wallner [101] discussed a Lagrangian form of the energy-momentum of some exact solutions in the teleparallel limit of PG theory.

We make a comparison between the possible values which reproduce the previous spacetimes of Schwarzschild and Reissner Nordström as shown in Table 1. Also, we give a comparison between the results of the total energy for taking into account Weitzenböck connection and for not taking into account Weitzenböck connection and the pervious results of the total energy for the same spacetime with arbitrary function as shown in Table 2.

\section{Acknowledgment}

This work is partially supported by the Egyptian Ministry of Scientific Research under project ID 24-2-12.

\section{References}

[1] T. Ortín, Gravity and Strings, Cambridge University Press, Cambridge, UK, 2004.

[2] C. Møller, "Tetrad fields and conservation laws in general relativity," in Proceedings of the International School of Physics "Enrico Fermi", C. Mller, Ed., Academic Press, London, UK, 1962.

[3] C. Møller, "Conservation laws in the tetrad theory of gravitation," in Proceedings of the Conference on Theory of Gravitation, Warszawa and Jablonna 1962, NORDITA Publications No. 136, Gauthier-Villars, Paris, France; PWN-Polish Scientific Publishers, Warszawa, Poland, 1964.

[4] C. Pellegrini and J. Plebański, Matematisk-fysiske skrifter udgivet af Det Kongelige Danske Videnskabernes Selskab, vol. 2, no. 4, 1963.

[5] F. W. Hehl, In ProceedIngs of the 6th School of Cosmology and Gravitation Course on spin, Torsion Rotation and Supergravity, Eric, Italy, Plenum, New York, NY, USA, 1979.

[6] F. W. Hehl, J. D. McCrea, E. W. Mielke, and Y. Neeman, "Metric-affine gauge theory of gravity: field equations, Noether identities, world spinors, and breaking of dilation invariance," Physics Reports, vol. 258, no. 1-2, pp. 1-171, 1995.

[7] K. Hayashi, "The gauge theory of the translation group and underlying geometry," Physics Letters B, vol. 69, no. 4, pp. 441444, 1977.

[8] K. Hayashi and T. Shirafuji, "New general relativity," Physical Review D, vol. 19, no. 12, pp. 3524-3553, 1979.

[9] K. Hayashi and T. Shirafuji, "Addendum to 'New general relativity"' Physical Review D, vol. 24, no. 12, pp. 3312-3314, 1981.

[10] F. I. Mikhail, M. I. Wanas, E. I. Lashin, and A. Hindawi, "Spherically symmetric solutions in Møller's tetrad theory of gravitation ," General Relativity and Gravitation, vol. 26, no. 9, pp. 869-876, 1994.

[11] N. L. Youssef and A. M. Sid-Ahmed, "Linear connections and curvature tensors in the geometry of parallelizable manifolds," Reports on Mathematical Physics, vol. 60, no. 1, pp. 39-53, 2007.

[12] M. Blagojevic and M. Vasilic, "Asymptotic symmetry and conserved quantities in the Poincare gauge theory of gravity," Classical and Quantum Gravity, vol. 5, no. 9, p. 1241, 1988. 
[13] T. Kawai, "Energy-momentum and angular momentum densities in gauge theories of gravity," Physical Review D, vol. 62, no. 10, p. 104014, 2000.

[14] T. Kawai, K. Shibata, and I. Tanaka, "Generalized equivalence principle in extended new general relativity," Progress of Theoretical Physics, vol. 104, no. 3, pp. 505-530, 2000.

[15] F. W. Hehl, J. Dermott McCrea, E. W. Mielke, and Y. Ne'eman, "Metric-affine gauge theory of gravity: field equations, Noether identities, world spinors, and breaking of dilation invariance," Physics Report, vol. 258, no. 1-2, pp. 1-171, 1995.

[16] Y. N. Obukhov, "Poincaré gauge gravity: selected topics," International Journal of Geometric Methods in Modern Physics, vol. 3, no. 1, p. 95, 2006.

[17] T. G. Lucas, Y. N. Obukhov, and J. G. Pereira, "Regularizing role of teleparallelism," Physical Review D, vol. 80, no. 6, p. 064043 , 2009.

[18] R. Aldrovandi, L. C. T. Guillen, J. G. Pereira, and K. H. Vu, "Bringing together gravity and the quanta," in Proceedings of the Albert Einstein Century International Conference, Paris, France, July 2005, [gr-qc/0603122].

[19] Y. N. Obukhov, J. G. Pereira, and G. F. Rubilar, "On the energy transported by exact plane gravitational-wave solutions," Classical and Quantum Gravity, vol. 26, no. 21, p. 215014, 2009.

[20] E. C. G. Sudarshan and N. Mukunda, Classical Dynamics: A Modern Perspective, John Wiley and Sons, New York, NY, USA, 1974.

[21] C. Itzykson and J. Zuber, Quantum Field Theory, McGraw-Hill, New York, NY, USA, 1980.

[22] H. Goldstein, Classical Mechanics, Addison-Wesley, Massachusetts, Mass, USA, 1980.

[23] L. B. Szabados, "Quasi-local energy-momentum and angular momentum in GR: a review article," Living Reviews in Relativity, vol. 7, p. 1, 2004.

[24] J. M. Nester, "General pseudotensors and quasilocal quantities," Classical and Quantum Gravity, vol. 21, no. 3, p. S261, 2004.

[25] J. W. Maluf, "Hamiltonian formulation of the teleparallel description of general relativity," Journal of Mathematical Physics, vol. 35, no. 1, pp. 335-343, 1994.

[26] J. Schwinger, "Quantized gravitational field," Physical Review, vol. 130, no. 3, pp. 1253-1258, 1963.

[27] J. W. Maluf, "Localization of energy in general relativity," Journal of Mathematical Physics, vol. 36, no. 8, pp. 4242-4247, 1995.

[28] J. W. Maluf, J. F. da Rocha-neto, T. M. L. Toribio, and K. H. Castello-Branco, "Energy and angular momentum of the gravitational field in the teleparallel geometry," Physical Review $D$, vol. 65, no. 12, p. 124001, 2002.

[29] G. W. Gibbons, M. J. Perry, and C. N. Pope, "The first law of thermodynamics for Kerr-anti-de Sitter black holes," Classical and Quantum Gravity, vol. 22, no. 9, p. 1503, 2005.

[30] A. Ashtekar and A. Magnon, "Asymptotically anti-de Sitter space-times," Classical and Quantum Gravity, vol. 1, no. 4, p. L39, 1984.

[31] A. Ashtekar and S. Das, "Asymptotically anti-de Sitter spacetimes: conserved quantities," Classical and Quantum Gravity, vol. 17, no. 2, p. L17, 2000.

[32] T. Regge and C. Teitelboim, "Role of surface integrals in the Hamiltonian formulation of general relativity," Annals of Physics, vol. 88, no. 1, pp. 286-318, 1974.

[33] L. F. Abbott and S. Deser, "Charge definition in non-abelian gauge theories,” Nuclear Physics, vol. 88, p. 286, 1974.
[34] S. Deser, I. Kanik, and B. Tekin, "Conserved charges of higher D Kerr-AdS spacetimes," Classical and Quantum Gravity, vol. 22, no. 17, pp. 3383-3389, 2005.

[35] S. Deser and B. Tekin, "New energy definition for highercurvature gravities," Physical Review D, vol. 75, no. 8, Article ID 084032, 2007.

[36] G. W. Gibbons, S. W. Hawking, G. T. Horowitz, and M. J. Perry, "Positive mass theorems for black holes," Communications in Mathematical Physics, vol. 88, no. 3, pp. 295-308, 1983.

[37] G. W. Gibbons, C. M. Hull, and N. P. Warner, "The stability of gauged supergravity," Nuclear Physics B, vol. 218, no. 1, pp. 173190, 1983.

[38] V. Iyer and R. M. Wald, "Some properties of the Noether charge and a proposal for dynamical black hole entropy ", Physical Review D, vol. 50, no. 2, pp. 846-864, 1994.

[39] R. Cai and L. Cao, "Conserved charges in even dimensional asymptotically locally anti-de Sitter space-times," Journal of High Energy Physics, vol. 603, no. 3, p. 83, 2006.

[40] S. Hollands, A. Ishibashi, and D. Marolf, "Comparison between various notions of conserved charges in asymptotically AdS spacetimes," Classical and Quantum Gravity, vol. 22, no. 14, p. 2881, 2005.

[41] R. M. Wald and A. Zoupas, "General definition of "conserved quantities" in general relativity and other theories of gravity," Physical Review D, vol. 61, no. 8, Article ID 084027, 2000.

[42] I. M. Anderson and C. G. Torre, "Asymptotic conservation laws in classical field theory," Physical Review Letters, vol. 77, no. 20, pp. 4109-4113, 1996.

[43] G. Barnich and F. Brandt, "Covariant theory of asymptotic symmetries, conservation laws and central charges "' Nuclear Physics B, vol. 633, no. 1-2, pp. 3-82, 2002.

[44] J. Katz, J. Bicak, and D. Lynden-Bell, "Relativistic conservation laws and integral constraints for large cosmological perturbations," Physical Review D, vol. 55, no. 10, pp. 5957-5969, 1997.

[45] N. Deruelle and J. Katz, "On the mass of a Kerr-anti-de Sitter spacetime in D dimensions ," Classical and Quantum Gravity, vol. 22, no. 2, p. 421, 2005.

[46] A. N. Aliev, "Electromagnetic properties of Kerr-anti-de Sitter black holes," Physical Review D, vol. 75, no. 8, Article ID 084041, 2007.

[47] B. Julia and S. Silva, "Currents and superpotentials in classical gauge-invariant theories: I. Local results with applications to perfect fluids and general relativity," Classical and Quantum Gravity, vol. 15, no. 8, p. 2173, 1998.

[48] Y. Obukhov and G. F. Rubilar, "Scalar $\Lambda \mathrm{N}$ and $\Lambda \Lambda$ interaction in a chiral unitary approach," Physical Review D, vol. 74, no. 6, Article ID 064002, 2006.

[49] S. Silva, "On superpotentials and charge algebras of gauge theories," Nuclear Physics B, vol. 558, no. 1-2, pp. 391-415, 1999.

[50] B. Julia and S. Silva, "Currents and superpotentials in classical gauge theories: II. Global aspects and the example of affine gravity," Classical and Quantum Gravity, vol. 17, no. 22, p. 4733, 2000.

[51] R. Aros, M. Contreras, R. Olea, R. Troncoso, and J. Zanelli, "Conserved charges for gravity with locally anti-de sitter asymptotics," Physical Review Letters, vol. 84, no. 8, pp. 1647$1650,2000$.

[52] R. Aros, M. Contreras, R. Olea, R. Troncoso, and J. Zanelli, "Conserved charges for even dimensional asymptotically AdS gravity theories," Physical Review D, vol. 62, no. 4, Article ID 044002, 2000. 
[53] M. Henningson and K. Skenderis, "The holographic Weyl anomaly," Journal of High Energy Physics, vol. 9807, no. 7, p. 23, 1998.

[54] V. Balasubramanian and P. Kraus, "A stress tensor for anti-de sitter gravity," Communications in Mathematical Physics, vol. 208, no. 2, pp. 413-428, 1999.

[55] G. Barnich and G. Compeere, "Generalized Smarr relation for Kerr-AdS black holes from improved surface integrals," Physical Review D, vol. 71, no. 4, Article ID 044016, 2005.

[56] M. Banados, R. Olea, and S. Theisen, "Counterterms and dual holographic anomalies in CS gravity," Journal of High Energy Physics, vol. 510, p. 67, 2005.

[57] R. Olea, "Regularization of odd-dimensional AdS gravity: kounterterms," Journal of High Energy Physics, vol. 704, p. 73, 2007.

[58] G. G. L. Nashed, "Total conserved charges of Kerr-NUT spacetimes using Poincaré version of teleparallel equivalent of general relativity," European Physical Journal C, vol. 72, no. 4, pp. 1-14, 2012.

[59] P. Baekler and F. W. Hehl, "Rotating black holes in metric-affine gravity," International Journal of Modern Physics D, vol. 15, no. 5, p. 635, 2006.

[60] J. M. Nester, F. H. Ho, and C. M. Chen, "Quasilocal center-ofmass for teleparallel gravity," in Proceedings of the 10th Marcel Grossman Meeting, J. M. Nester, Ed., vol. 33, p. 910, Journal of Mathematical Physics, 1992.

[61] R. S. Tung and J. M. Nester, "The quadratic spinor Lagrangian is equivalent to the teleparallel theory," Physical Review D, vol. 60, no. 2, Article ID 021501, 1999.

[62] V. C. de Andrade and J. G. Pereira, "Gravitational Lorentz force and the description of the gravitational interaction " Physical Review D, vol. 56, no. 8, pp. 4689-4695, 1997.

[63] Y. M. Cho, "Einstein Lagrangian as the translational Yang-Mills Lagrangian ," Physical Review D, vol. 14, no. 10, pp. 2521-2525, 1976.

[64] F. Gronwald, "Metric-affine gauge theory of gravity: I. Fundamental structure and field equations," International Journal of Modern Physics D, vol. 6, no. 3, p. 263, 1997.

[65] U. Muench, Über teleparallele Gravitationstheorien [M.S. thesis], University of Cologne, Cologne, Germany, 1997.

[66] D. K. Wise, "Symmetric space cartan connections and gravity in three and four dimensions," Symmetry, Integrability and Geometry, vol. 5, p. 80, 2009.

[67] V. C. de Andrade and J. G. Pereira, "Gravitational Lorentz force and the description of the gravitational interaction ", Physical Review D, vol. 56, no. 8, pp. 4689-4695, 1997.

[68] R. Tresguerres, "Translations and dynamics," International Journal of Geometric Methods in Modern Physics, vol. 5, no. 6, p. 905 , 2008.

[69] Y. N. Obukhov and J. G. Pereira, "Metric-affine approach to teleparallel gravity," Physical Review D, vol. 67, no. 4, Article ID 044016, 2003.

[70] Y. N. Obukhov, "Plane waves in metric-affine gravity," Physical Review D, vol. 73, no. 2, Article ID 024025, 2006.

[71] Y. N. Obukhov, G. F. Rubilar, and J. G. Pereira, "Conserved currents in gravitational models with quasi-invariant Lagrangians: application to teleparallel gravity," Physical Review D, vol. 74, no. 10, Article ID 104007, 2006.

[72] Y. N. Obukhov and J. G. Pereira, "Lessons of spin and torsion: reply to 'Consistent coupling to Dirac fields in teleparallelism," Physical Review D, vol. 69, no. 12, Article ID 128502, 2004.
[73] D. Puetzfeld, "An exact-plane fronted wave solution in metricaffine gravity," in Exact Solutions and Scalar Field in Gravity: Recent Developments, A. Macias, J. Cervantes-Cota, and C. Lammerzahl, Eds., pp. 141-151, Kluwer, Dordrecht, The Netherlands, 2001.

[74] A. Garcia, A. Macias, D. Puetzfeld, and J. Socorro, "Planefronted waves in metric-affine gravity," Physical Review D, vol. 62, no. 4, Article ID 044021, 2000.

[75] A. D. King and D. Vassiliev, "Torsion waves in metric-affine field theory," Classical and Quantum Gravity, vol. 18, no. 12, pp. 23172329, 2001.

[76] J. D. Brown and J. W. York, "Quasilocal energy and conserved charges derived from the gravitational action," Physical Review $D$, vol. 47, no. 4, pp. 1407-1419, 1993.

[77] V. Pasic and D. Vassiliev, "Pp-waves with torsion and metricaffine gravity," Classical and Quantum Gravity, vol. 22, no. 19, pp. 3961-3975, 2005.

[78] D. Vassiliev, "Pseudoinstantons in metric-affine field theory," General Relativity and Gravitation, vol. 34, no. 8, pp. 1239-1265, 2002.

[79] D. Vassiliev, "Quadratic metric-affine gravity," Annalen der Physik (Leipzig), vol. 14, no. 4, pp. 231-252, 2005.

[80] H. T. Nieh, “A torsional topological invariant," International Journal of Modern Physics A, vol. 22, no. 29, p. 5237, 2007.

[81] R. Jackiw and S.-Y. Pi, "Chern-Simons modification of general relativity," Physical Review D, vol. 68, no. 10, Article ID 104012, 2003.

[82] M. B. Cantcheff, "Einstein-Cartan formulation of ChernSimons Lorentz-violating gravity," Physical Review D, vol. 78, no. 2, Article ID 025002, 2008.

[83] E. W. Mielke, "Einsteinian gravity from a topological action," General Relativity and Gravitation, vol. 40, no. 6, pp. 1311-1325, 2008.

[84] E. W. Mielke, “Topologically modified teleparallelism, passing through the Nieh-Yan functional," Physical Review D, vol. 80, no. 6, Article ID 067502, 2009.

[85] Ph. Freud, "Uber die ausdrucke der gesamtenergie und des gesamtimpulses eines materiellen systems in der allgemeinen relativitatstheorie," Annals of Mathematics, vol. 40, no. 2, pp. 417-419, 1939.

[86] J. M. Nester, "A new gravitational energy expression with a simple positivity proof," Physics Letters A, vol. 83, no. 6, pp. 241242, 1981.

[87] J. Socorro, A. Macias, and F. W. Hehl, "Computer algebra in gravity: reduce-excalc programs for (non-) Riemannian spacetimes. I," Computer Physics Communications, vol. 115, no. 2-3, pp. 264-283, 1998.

[88] G. G. L. Nashed and T. Shirafuji, "Reissner-Nordström spacetime in the tetrad theory of gravitation," International Journal of Modern Physics D, vol. 16, no. 1, p. 65, 2007.

[89] G. G. L. Nashed, "Vacuum non singular black hole solutions in tetrad theory of gravitation," General Relativity and Gravitation, vol. 34, no. 7, pp. 1047-1058, 2002.

[90] T. Shirafuji, G. G. L. Nashed, and K. Hayashi, "Energy of general spherically symmetric solution in the tetrad theory of gravitation," Progress of Theoretical Physics, vol. 95, no. 3, pp. 665-678, 1996.

[91] G. G. L. Nashed, "General spherically symmetric nonsingular black hole solutions in a teleparallel theory of gravitation," Physical Review D, vol. 66, no. 6, Article ID 064015, 2002. 
[92] R. Arnowitt, S. Deser, and C. W. Misner, "The dynamics of general relativity," in Gravitation: An Introduction to Current Research, L. Witten, Ed., Wiley, New York, NY, USA, 1962.

[93] G. G. L. Nashed, "Reissner-Nordström solutions and energy in teleparallel theory," Modern Physics Letters A, vol. 21, no. 29, p. 2241, 2006.

[94] G. G. L. Nashed, "Energy of spherically symmetric space-times on regularizing teleparallelism," International Journal of Modern Physics A, vol. 25, no. 14, p. 2883, 2010.

[95] G. W. Gibbons and S. W. Hawking, "Action integrals and partition functions in quantum gravity," Physical Review $D$, vol. 15, no. 10, pp. 2752-2756, 1977.

[96] S. W. Hawking, "Breakdown of predictability in gravitational collapse," Physical Review D, vol. 14, no. 10, pp. 2460-2473, 1976.

[97] S. W. Hawking, in GeneralRelativity, S. W. Hawking and W. Israel, Eds., Cambridge University Press, Cambridge, UK, 1979.

[98] W. Kim, E. J. Son, and M. Yoon, “Thermodynamic similarity between the noncommutative Schwarzschild black hole and the Reissner-Nordström black hole," Journal of High Energy Physics, vol. 2008, no. 4, p. 42, 2008.

[99] M. M. Akbar, "Entropy corrections for Schwarzschild and Reissner-Nordström black holes," Classical and Quantum Gravity, vol. 21, no. 6, p. 1383, 2004.

[100] M. Blagojević, "Hamiltonian structure and gauge symmetries of Poincaré gauge theory," Annalen der Physik, vol. 10, no. 5, pp. 367-391, 2001.

[101] E. W. Mielke and R. P. Wallner, "Mass and spin of double dual solutions in Poincaré gauge theory," Nuovo Cimento B, vol. 101, no. 5, pp. 607-624, 1988. 

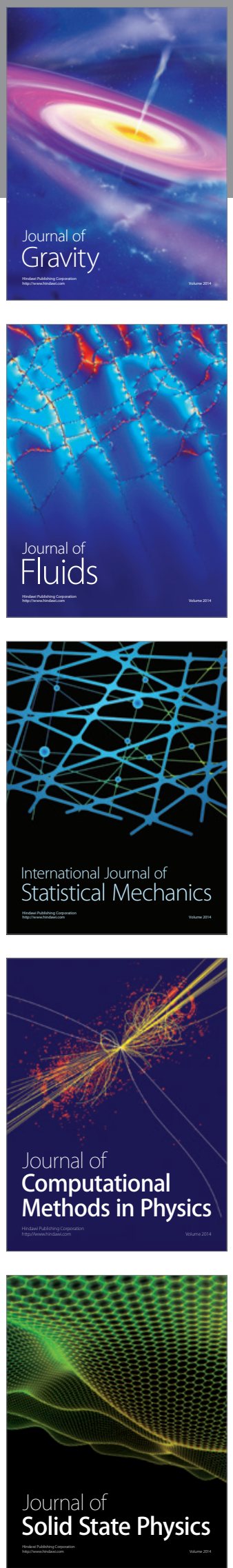

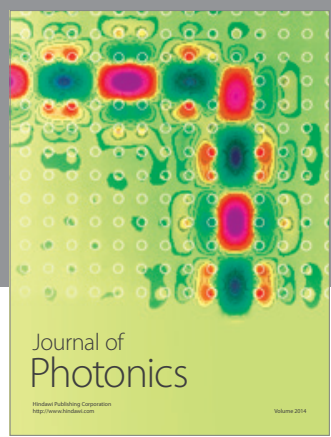

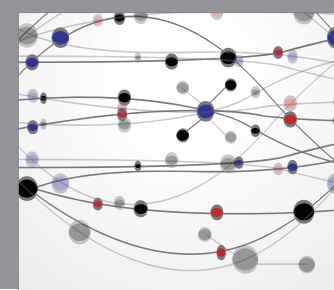

The Scientific World Journal

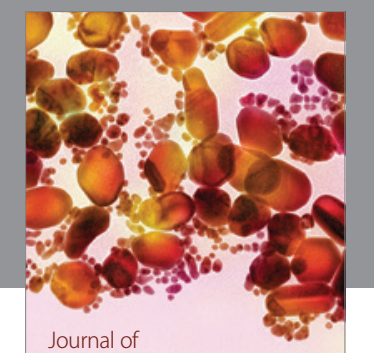

Soft Matter
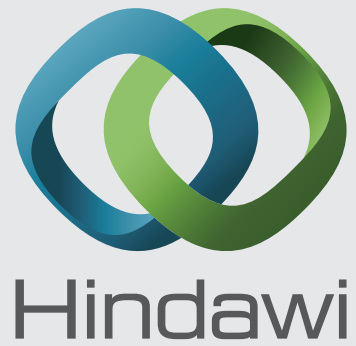

Submit your manuscripts at

http://www.hindawi.com
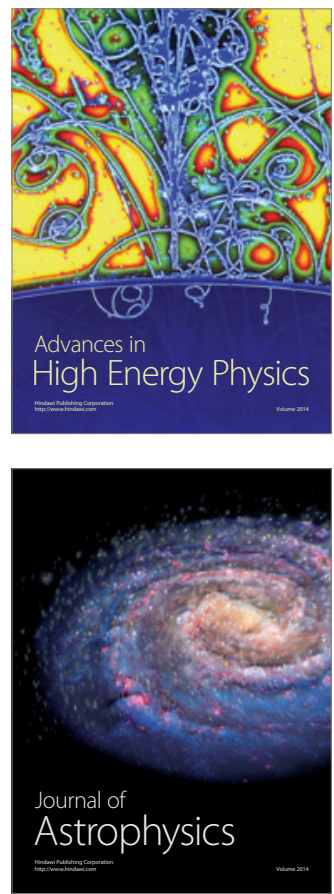
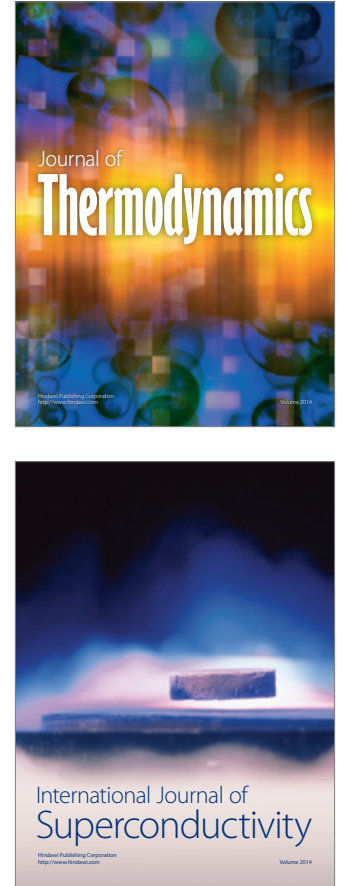
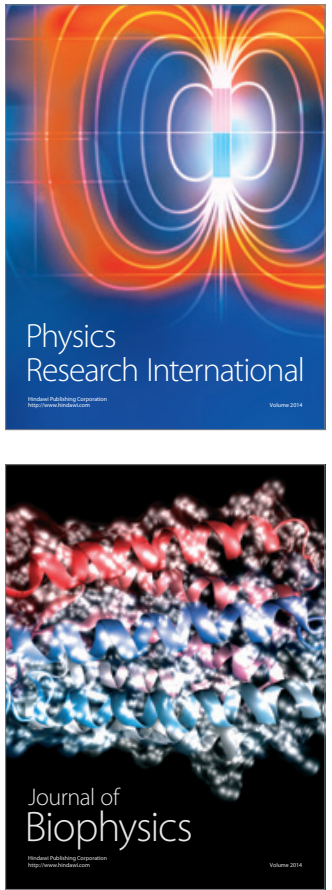
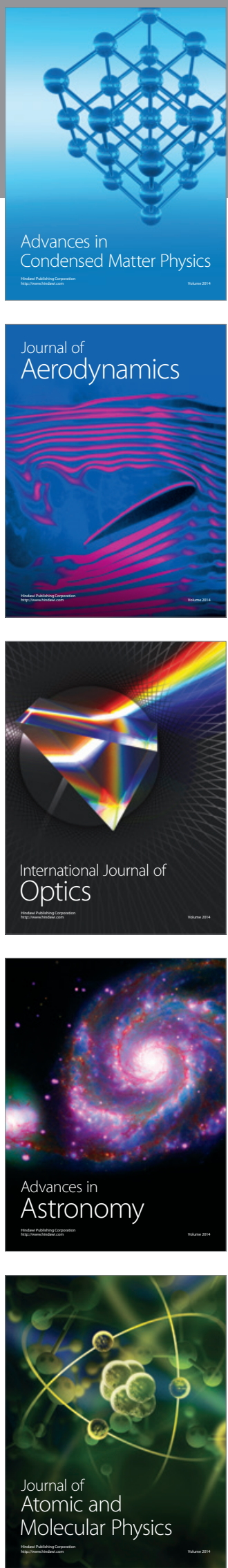